University of Nebraska - Lincoln

DigitalCommons@University of Nebraska - Lincoln

Robert Streubel Papers

Research Papers in Physics and Astronomy

7-19-2019

\title{
Reconfigurable ferromagnetic liquid droplets
}

Xubo Liu

Noah Kent

Alejandro Ceballos

Robert Streubel

Yufeng Jiang

See next page for additional authors

Follow this and additional works at: https://digitalcommons.unl.edu/physicsstreubel

Part of the Atomic, Molecular and Optical Physics Commons, Condensed Matter Physics Commons, and the Other Physics Commons

This Article is brought to you for free and open access by the Research Papers in Physics and Astronomy at DigitalCommons@University of Nebraska - Lincoln. It has been accepted for inclusion in Robert Streubel Papers by an authorized administrator of DigitalCommons@University of Nebraska - Lincoln. 


\section{Authors}

Xubo Liu, Noah Kent, Alejandro Ceballos, Robert Streubel, Yufeng Jiang, Yu Chai, Paul Y. Kim, Joe Forth, Frances Hellman, Shaowei Shi, Dong Wang, Brett A. Helms, Paul D. Ashby, Peter Fischer, and Thomas P. Russell 


\title{
Reconfigurable ferromagnetic liquid droplets
}

\author{
Xubo Liu ${ }^{1,2}$, Noah Kent ${ }^{2,3}$, Alejandro Ceballos', \\ Robert Streubel $^{2}$, Yufeng Jiang ${ }^{2,5}$, Yu Chai ${ }^{2,4,6}$, \\ Paul Y. Kim², Joe Forth², Frances Hellman²,7, \\ Shaowei Shi ${ }^{1}$, Dong Wang ${ }^{1,8}$, Brett A. Helms ${ }^{2,6}$, \\ Paul D. Ashby ${ }^{2,6}$, Peter Fischer ${ }^{2,3}$, and \\ Thomas P. Russell $1,2,9,10 *$
}

1 Beijing Advanced Innovation Center for Soft Matter Science and Engineering, Beijing University of Chemical Technology, Beijing 100029, China.

2 Materials Sciences Division, Lawrence Berkeley National Laboratory, Berkeley, CA 94720, USA.

3 Physics Department, University of California, Santa Cruz, Santa Cruz, CA 95064, USA.

4 Department of Materials Science and Engineering, University of California, Berkeley, Berkeley, CA 94720, USA.

5 Department of Applied Science and Technology, University of California, Berkeley, Berkeley, CA 94720, USA.

6 The Molecular Foundry, Lawrence Berkeley National Laboratory, Berkeley, CA 94720, USA.

7 Physics Department, University of California, Berkeley, Berkeley, CA 94720, USA.

8 State Key Laboratory of Organic-Inorganic Composites, Beijing University of Chemical Technology, Beijing 100029, China.

9 Polymer Science and Engineering Department, University of Massachusetts, Amherst, MA 01003, USA.

10 WPI-Advanced Institute for Materials Research (WPI-AIMR), Tohoku University, Sendai 980-8577, Japan.

* Corresponding author - T.P. Russell, email tom.p.russell@gmail.com

Published in Science 365, 264-267 (19 July 2019).

DOI: $10.1126 /$ science.aaw8719

Copyright (C) 2019 by the Authors; exclusive licensee American Association for the Advancement of Science. Used by permission.

Submitted 31 January 2019; accepted 24 May 2019 


\begin{abstract}
Solid ferromagnetic materials are rigid in shape and cannot be reconfigured. Ferrofluids, although reconfigurable, are paramagnetic at room temperature and lose their magnetization when the applied magnetic field is removed. Here, we show a reversible paramagnetic-to-ferromagnetic transformation of ferrofluid droplets by the jamming of a monolayer of magnetic nanoparticles assembled at the water-oil interface. These ferromagnetic liquid droplets exhibit a finite coercivity and remanent magnetization. They can be easily reconfigured into different shapes while preserving themagnetic properties of solid ferromagnets with classic north-south dipole interactions. Their translational and rotational motions can be actuated remotely and precisely by an external magnetic field, inspiring studies on active matter, energy-dissipative assemblies, and programmable liquid constructs.
\end{abstract}

Ferromagnetic materials are generally solids with a fixed shape. Reconfigurable magnetic materials are known, such as ferrofluids [dispersions of magnetic nanoparticles (MNPs) in carrier fluids], but they are paramagnetic and lose magnetization once the external magnetic field is removed $(1,2)$. Ferrofluids exhibit interesting properties and have found use, for example, as magnetic seals, but their inability to retain magnetization limits their broader application. The transformation of a ferrofluid into a ferromagnetic material can be realized by lowering the temperature or increasing the viscosity, at which Brownian motion of the MNPs is suppressed. Here, we show a simple means to effect this transformation by the in situ formation and interfacial jamming of MNP-surfactants.

We immersed an aqueous dispersion of carboxylated 22-nm-diameter MNPs $\left(\mathrm{Fe}_{3} \mathrm{O}_{4}-\mathrm{CO}_{2} \mathrm{H}\right)$ in a solution of amine-modified polyhedral oligomeric silsesquioxane (POSS- $\mathrm{NH}_{2}$ ) intoluene. The POSS- $\mathrm{NH}_{2}$, itself a surfactant, assembles at the interface and electrostatically interacts with the MNPs, anchoring a well-defined number of POSS- $\mathrm{NH}_{2}$ to the MNPs, converting the MNPs into MNP-surfactants. When the droplet shape changes, the interfacial area increases, and additional MNP-surfactants form and assemble at the interface. The droplet proceeds to reshape itself to minimize the interfacial area and, thereby, the free energy of the system, but the MNP-surfactants are compressed and jam, locking in the deformed shape $(3,4)$ while remaining magnetized even without an external field.

Magnetic hysteresis loops of $\mathrm{Fe}_{3} \mathrm{O}_{4}-\mathrm{CO}_{2} \mathrm{H}$ ferrofluid droplets (Fig. $1 \mathrm{~A})$, measured by a vibrating sample magnetometer, show a saturation magnetization $\left(M_{\mathrm{s}}\right)$ that depends on the total number of MNPs 
in the droplets, as well as a vanishing coercive field $\left(H_{\mathrm{c}}\right)$ and remanent magnetization $\left(M_{\mathrm{r}}\right)$. By adding POSS- $\mathrm{NH}_{2}$ ligands to the toluene, MNP-surfactants form at the interface. Increasing the concentration of the MNPs in the droplet or decreasing $\mathrm{pH}$ increases the coverage of the interface byMNP-surfactants, reducing the interfacial tension (Figs. S1, A and B). With sufficient surface coverage, the MNP-surfactants jam, and the ferrofluid droplet transforms into a ferromagnetic liquid droplet. The magnetic hysteresis loops of identical ferrofluid droplets with and without the jammed interfacial assemblies of MNP-surfactants are shown in Fig. 1A. For both, $M_{s}$ is the same because the total number of MNPs is identical, but $M_{\mathrm{r}} \sim 1.89 \times 10^{-8} \mathrm{~A} \mathrm{~m}^{2}$ and $\mathrm{H}_{\mathrm{c}} \sim 7.2 \mathrm{kA} \mathrm{m}^{-1}$ for the ferromagnetic liquid droplet, demonstrating their ferromagnetic character. The jammed, interfacial assemblies of the MNP-surfactants are disordered and have a mechanical rigidity that suppresses thermal fluctuations characteristic of isolated MNPs. The jammed MNPs no longer freely rotate. The spatial separation between adjacent MNP-surfactants is $<5 \mathrm{~nm}$, which, combined with the orientation of the dipole magnetization in the MNPs, enhances the thermal stability of the magnetization and transforms the droplet surface into a ferromagnetic layer, similar to a fixed assembly of MNPs $(5,6)$. When the field is removed, the moment of the ferromagnetic liquid droplet remains until the droplet is exposed to a field exceeding the switching field, whereupon the droplet is remagnetized. Reshaping the droplet by other external fields or reducing the binding energy of the MNPsurfactants will also unjam the MNP-surfactants ( $M_{\mathrm{r}}$ and $H_{\mathrm{c}}$ vanish), providing further routes to control the magnetization. This ability to manipulate the magnetization further distinguishes ferromagnetic liquid droplets from ferrofluids and common ferromagnetic materials.

If the MNP-surfactant assembly is not jammed, no hysteresis is observed (Fig. 1B). To produce droplets with an unjammed assembly, the surface coverage of the droplets with the MNP-surfactants is varied from $\sim 7$ to $\sim 20 \%$ (Fig. S1C) by changing the concentrations of the MNPs and POSS- $\mathrm{NH}_{2}$ and the pH. In Fig. 1B, variations in $M_{\mathrm{s}}$ arise from differences in the total number of MNPs in each droplet. With full MNP-surfactant coverage-for example, single droplets of $\left[\mathrm{Fe}_{3} \mathrm{O}_{4}^{-}\right.$ $\mathrm{CO}_{2} \mathrm{H}$ MNPs] $=0.5 \mathrm{~g} \mathrm{~L}^{-1}$ at $\mathrm{pH} 4.5$ in toluene containing [POSS- $\mathrm{NH}_{2}$ ] = 1.o $\mathrm{g} \mathrm{L}^{-1}$-the interfacial assembly jams, and a typical ferromagnetic 
A

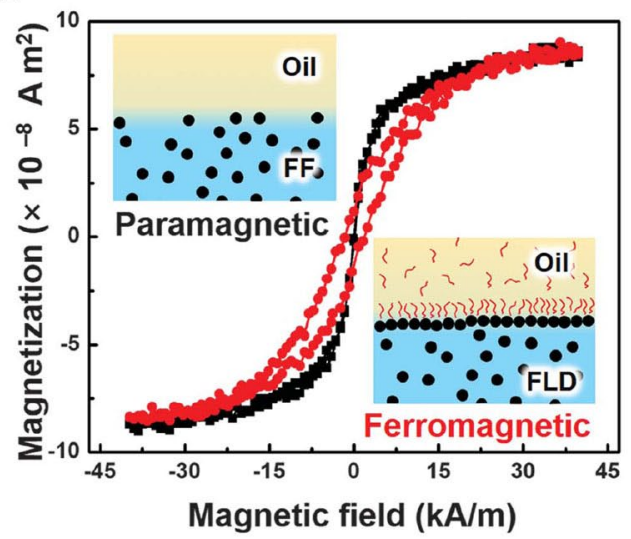

C

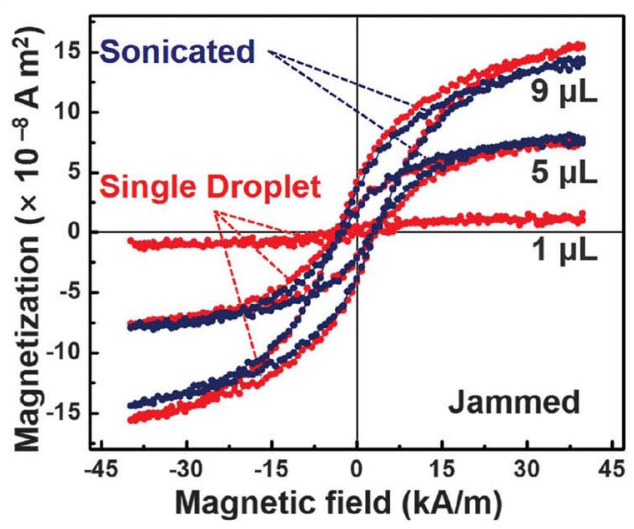

B

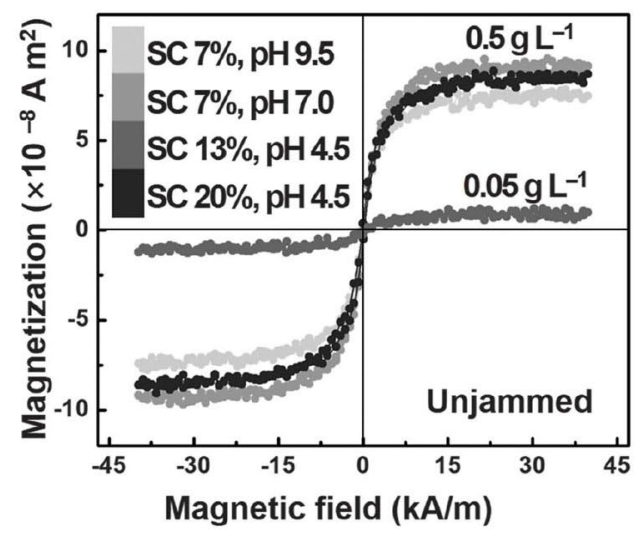

D

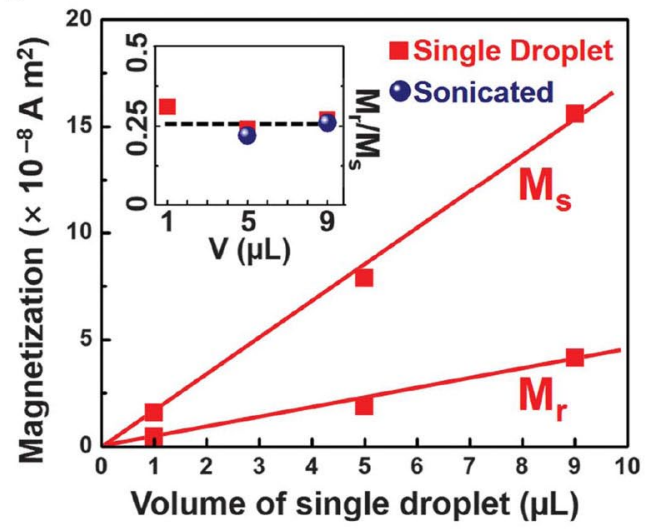

Fig. 1. Tunable transformation of a paramagnetic FF into an FLD by the interfacial jamming of MNP-surfactants.

A) Magnetic hysteresis loops of droplets with (red line) and without (black line) an interfacial layer of jammed MNP-surfactants measured with a vibrating sample magnetometer. Two schematics of aqueous FF and FLD, containing $\mathrm{Fe}_{3} \mathrm{O}_{4}-\mathrm{CO}_{2} \mathrm{H}$ MNPs $\left(0.5 \mathrm{~g} \mathrm{~L}^{-1}\right)$ at $\mathrm{pH} 4.5$, immersed in toluene without and with POSS- $\mathrm{NH}_{2} \mathrm{li}$ gands $\left(1.0 \mathrm{~g} \mathrm{~L}^{-1}\right)$.

B) Hysteresis loops of individual 5-mL aqueous droplets, with $0.5 \mathrm{~g} \mathrm{~L}^{-1}$ and $0.05 \mathrm{~g}$ $\mathrm{L}^{-1}$ of $\mathrm{Fe}_{3} \mathrm{O}_{4}-\mathrm{CO}_{2} \mathrm{H}$ MNPs at different $\mathrm{pH}$, immersed in $0.01 \mathrm{~g} \mathrm{~L}^{-1}$ ligand solution. Surface coverage (SC) of droplets is $\sim 7$ to $\sim 20 \%$ where MNPS assemblies are not jammed.

C) Hysteresis loops of single, jammed aqueous droplets with $0.5 \mathrm{~g} \mathrm{~L}^{-1}$ of MNPs at $\mathrm{pH}$ 4.5 immersed in a $1.0 \mathrm{~g} \mathrm{~L}^{-1}$ solution of POSS $-\mathrm{NH}_{2}$ in toluene and hysteresis loops of the same system after being sonicated (figs. S1 and S2).

D) $M_{r}$ and $M_{s}$ of the droplets as a function of droplet volume. In the inset, the remanence ratio $M_{r} / M_{s}$ as a function of initial droplet volume (single droplet or droplet sonicated into multiple smaller droplets) remains constant at $\sim 0.25$. FF, ferrofluid; FLD, ferromagnetic liquid droplet. 
hysteresis loop is seen (Fig. 1C). Hysteresis loops were measured for single droplets with different volumes, and the same droplets sonicated into numerous smaller droplets. This preserves the total volume (summed over all droplets) of the MNP dispersions but increases the surface-to-volume ratio $(S / V)$ by two orders of magnitude (Fig. S2D). $M_{\mathrm{s}}$ and $M_{\mathrm{r}}$ scale linearly with the total volume (total number of MNPs), whereas $H_{\mathrm{c}}$ remains constant (Fig. 1C). For a given total volume, $M_{\mathrm{r}}$ is independent of $S / V$ with largely varying droplet sizes. The mean separation distance between the dispersed MNPs is $\sim 350 \mathrm{~nm}$, too large for dipolar coupling. For comparison, discs that are $100 \mathrm{~nm}$ in diameter and $10 \mathrm{~nm}$ thick and have much larger saturation magnetizations are completely uncorrelated when the separation distance is $>60 \mathrm{~nm}$ (7). The MNPs dispersed in the droplet freely diffuse, yet a strong coupling and correlation of the dispersed MNPs to those jammed at the interface is evident, and the liquid droplets behave like solid magnets. Furthermore, the ratio of $\mathrm{M}_{\mathrm{r}} / \mathrm{M}_{\mathrm{s}}$ for the ferromagnetic liquid droplets is 0.25 , independent of droplet volume, which is the same as that for frozen ferrofluids at $4.5 \mathrm{~K}$ and fixed assemblies of $\mathrm{Fe}_{3} \mathrm{O}_{4} \mathrm{MNPs}(8-10)$. Consequently, ferromagnetic liquid droplets have a similar energy barrier to overcome during magnetization reversal as their frozen or solid counterparts. Therefore, ferromagnetic liquid droplets have the magnetic properties of a solid.

All-liquid printing (11-13) and microfluidics (14) were used to produce ferromagnetic liquid cylinders with a 2:1 aspect ratio (Fig. S3). A nonmagnetized ferromagnetic liquid cylinder was transferred to a toluene/ $\mathrm{CCl}_{4}$ density gradient in which the cylinder descended until buoyant (Fig. S4, A and B). The axis of the cylinder and an insulated solenoid were aligned (Fig. 2A), and a magnetic field of 1 to $\sim 2 \mathrm{kA} \mathrm{m}^{-1}$ (Fig. 2B) applied to the solenoid pulled the ferromagnetic liquid cylinder into the solenoid. The cylinder reached the solenoid in $30 \mathrm{~s}$ at a speed of $\sim 1.1 \times 10^{-4} \mathrm{~m} \mathrm{~s}^{-1}$ and stopped after fully entering the solenoid because of the absence of drag forces.

The displacement, $\Delta d$ (in units of the droplet length, $L$ ), allows comparison of different ferromagnetic liquid cylinders because the drag force varies linearly with length, which for a fixed cylinder radius corresponds to the volume and $M_{\mathrm{s}}$. Figure 2A shows the location and direction of the magnetic moment of the ferromagnetic liquid cylinder. The cylinder, magnetized only by the solenoid, moved inside the 

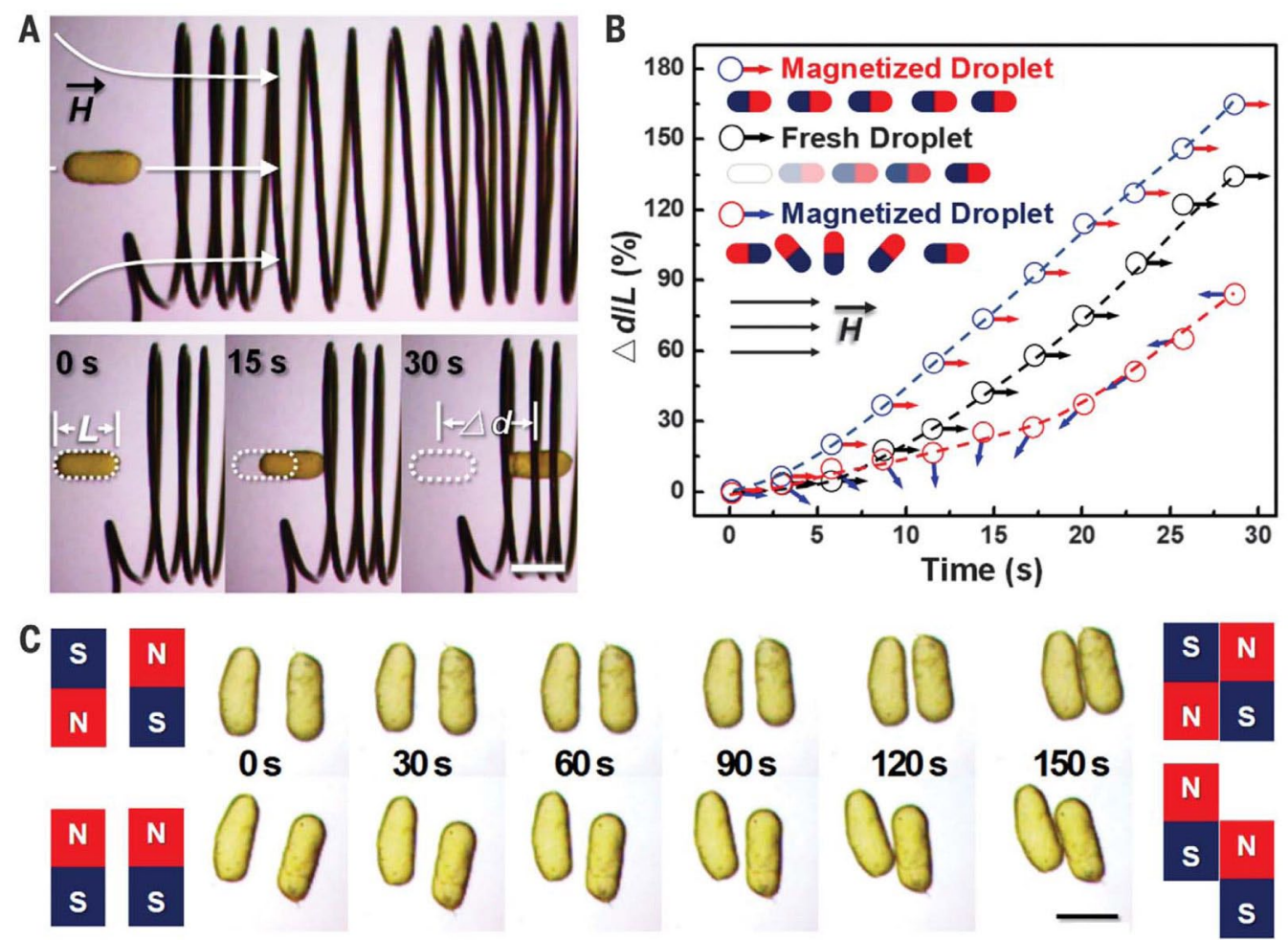

Fig. 2. Manipulating FLDs with magnetic dipole interactions.

A) A magnetized liquid cylinder is attracted by a magnetic field gradient, generated by the aluminum solenoid.

B) Displacement as a function of time for fresh, nonmagnetized liquid cylinder (black arrow), magnetized liquid cylinder with north pole facing the coil (red arrow), and magnetized liquid bar with south pole facing the coil (blue arrow). The ultimate velocity is determined by the field gradient and magnetic moment. The arrows indicate the orientation of the cylinder relative to the initial orientation.

C) Dipole interactions, $\mathrm{N}-\mathrm{S}$ attraction, $\mathrm{N}-\mathrm{N}$ and $\mathrm{S}-\mathrm{S}$ repulsion, between two magnetized liquid cylinders. Scale bars, $2 \mathrm{~mm}$.

solenoid (movie S1). This ferromagnetic liquid cylinder (now magnetized) was repositioned outside the solenoid, preserving the southnorth (S-N) pole orientation. With the same solenoid magnetic field strength ( 1 to $\sim 2 \mathrm{kA} \mathrm{m}^{-1}$ ), the cylinder now accelerates to the solenoid (Fig. 2B and movie S2). By reversing the field direction of the solenoid (movie S3), the magnetic moment of the ferromagnetic liquid cylinder and the solenoid field are in antiparallel alignment and should repel each other. However, initially a slight attraction is seen because of the free MNPs in the cylinder core; then, the cylinder rotates, aligning 
the moment of the jammed MNP-surfactants with the solenoid field, and is drawn into the solenoid (Fig. 2B). Considering the low velocity $\left(\mathrm{v}_{\max } \sim 1.1 \times 10^{-4} \mathrm{~m} \mathrm{~s}^{-1}\right)$ and corresponding low Reynolds number (Re $\sim 0.16$ ), the velocity of the ferromagnetic liquid cylinders can be expressed approximately as a function of time:

$$
v(t)=C \frac{m a}{b}\left(1-e^{-b t / m}\right)
$$

where $C$ is a dimensionless constant based on $R e$ and the shape of a liquid droplet, $a$ is the acceleration from the drag force $\left(a=F_{d} / m\right), m$ is the mass, and $b$ is the coefficient of viscous friction. The constant velocity at longer times, independent of the initial magnetization configuration (Fig. 2B), implies a constant solenoid field gradient (due to the same $M_{\mathrm{s}}$ ). Deviations from the linear relation in the early stage originate from viscous drag, the varying field gradient outside the solenoid, and the distinct initial magnetization configuration of the cylinder. Consequently, ferromagnetic liquid cylinders behave like solid magnets with $\mathrm{N}-\mathrm{N}, \mathrm{S}-\mathrm{S}$, and $\mathrm{N}-\mathrm{S}$ dipole interactions. Figure $2 \mathrm{C}$ shows that magnetized liquid cylinders, initially separated by $1 \mathrm{~mm}$, attract each other by such $\mathrm{N}-\mathrm{S}$ dipole interactions (movie $\mathrm{S} 4$ ).

A distinguishing feature of the ferromagnetic liquid droplets is reconfigurability. A 3-mL spherical ferromagnetic liquid droplet (1.4$\mathrm{mm}$ diameter) was drawn into a 1-mm-diameter glass capillary and then rapidly (after several seconds) ejected (Fig. 3, A and B, and movie S5). This transformed the spherical droplet into a cylinder (aspect ratio of 3:1). The interfacial area increased by 2.5 times, allowing more MNP-surfactants to form and jam, preserving the cylindrical shape. The ferromagnetic liquid droplet retained its ferromagnetic character, as evidenced by the rotation $\left(\sim 0.6 \mathrm{rad} \mathrm{s}^{-1}\right)$ in response to a rotating permanent magnetic field. The cylinder rotation is slightly less than that of the spherical droplet $\left(\sim 0.8 \mathrm{rad} \mathrm{s}^{-1}\right)$ because of the higher viscous drag force on the cylinder. The shape change can be reversed by tuning the binding energy, as shown in Fig. $3 \mathrm{C}$, in which the $\mathrm{pH}$ was increased from 4.5 to 9, allowing the MNP-surfactants to unjam and the droplet shape to revert to spherical. Magnetization is lost, but by decreasing the $\mathrm{pH}$, the MNP-surfactants rejam and the droplet transforms back to a ferromagnetic liquid droplet. Thus, the shape and magnetic state of the ferromagnetic liquid droplets are responsive. 


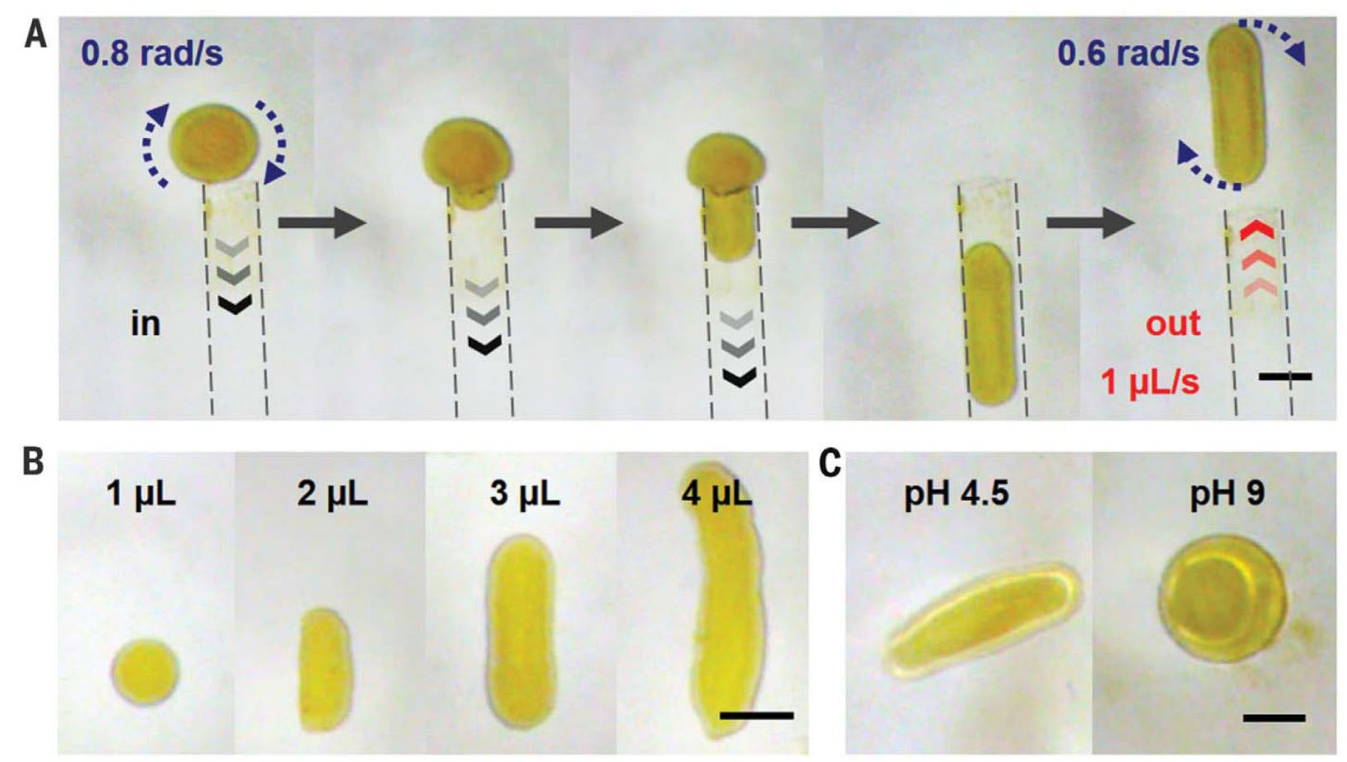

Fig. 3. Top view of deforming an FLD.

A) Reshaping spherical droplet into the cylinder by mechanically molding it with the glass capillary channel.

B) Droplets of different aspect ratios can be formed by using droplets with different volumes.

C) Reconfiguration of interfacial jamming and unjamming of MNP-surfactants by tuning the $\mathrm{pH}$ (for example, 4.5 and 9, respectively) of aqueous solution in the FLD cylinder. Scale bars, $1 \mathrm{~mm}$.

The necessity of the magnetic coupling between the interfacially jammed MNP-surfactants and the dispersed MNPs to generate ferromagnetic liquid droplets is shown by changing the nature of the jammed interfacial assembly. Two sets of ferrofluid droplets at a $\mathrm{pH}$ of 4.5 were placed in a mixture of toluene and tetrachloride carbon of equal density containing POSS- $\mathrm{NH}_{2}$ ligands: (i) ferrofluid droplets with carboxyl-functionalized 30-nm (22-nmcore) $\mathrm{Fe}_{3} \mathrm{O}_{4}$ MNPs (0.5 g L'-1) that form ferromagnetic liquid droplets and (ii) a mixture of nonmagnetic sodium carboxymethyl cellulose $\left(\mathrm{CMC}-\mathrm{CO}_{2} \mathrm{Na}\right)\left(0.5 \mathrm{~g} \mathrm{~L}^{-1}\right)$ with 30-nm $\mathrm{Fe}_{3} \mathrm{O}_{4}$-polyethylene glycol (PEG) (nonfunctionalized) MNPs (0.5 $\mathrm{g} \mathrm{L}^{-1}$ ), in which only the CMC interacts with the POSS- $\mathrm{NH}_{2}$. In both, nanoparticles jam at the interface (Fig. 4A), but in the first case they are ferromagnetic, whereas in the latter they are not ferromagnetic. Ferrofluid droplets of only PEG-functionalized $\mathrm{Fe}_{3} \mathrm{O}_{4} \mathrm{MNPs}\left(0.5 \mathrm{~g} \mathrm{~L}^{-1}\right)$ 

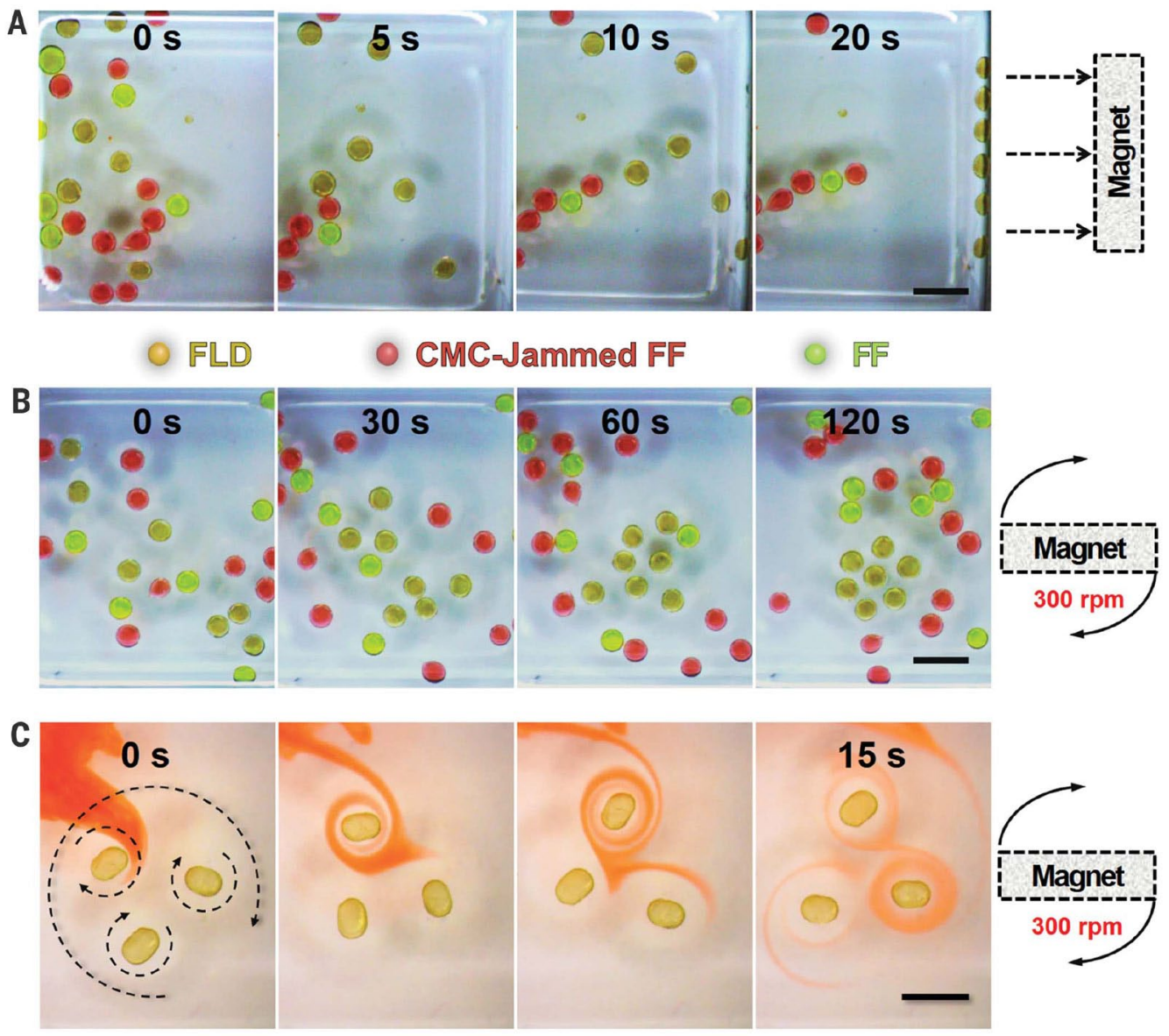

Fig. 4. Sorting FLDs by using static and rotational magnetic fields. Mixtures of FLDs comprising a shell of a jammed monolayer of MNP-surfactants and an FF core (dispersed carboxyl-functionalized iron oxide $\mathrm{NPs}_{1} \mathrm{Fe}_{3} \mathrm{O}_{4}-\mathrm{CO}_{2} \mathrm{H}$ ) (brownish spheres); FF droplet comprising a shell of jammed, nonmagnetic CMC surfactants (CMCSs) encapsulating an aqueous dispersion of PEG-coated iron oxide $\mathrm{NPs}\left(\mathrm{Fe}_{3} \mathrm{O}_{4}\right.$-PEG) (red spheres); $\mathrm{FF}$ droplet comprising an aqueous dispersion of $\mathrm{Fe}_{3} \mathrm{O}_{4}-\mathrm{PEG}$ NPs with no jammed monolayer at the droplet surface (bright green spheres). The separation of FLDs by using

A) a static bar magnet on the side of the container and

B) a bar magnet rotating under the container. [Rhodamine B in CMC-jammed FF] = $1 \mathrm{~g} \mathrm{~L}^{-1}$, [fluorescein sodium salt in FF] $=1 \mathrm{~g} \mathrm{~L}^{-1}$.

C) Visualization of the hydrodynamic vortex flow for an FLD ensemble in a rotating magnetic field using an oil-soluble dye. [Nile red in toluene] $=1 \mathrm{~g} \mathrm{~L}^{-1}$. Scale bars, $3 \mathrm{~mm}$. 
were also placed in the oil. The total number of MNPs in all the droplets was constant. A bar magnet attracts all droplets (Fig. 4A) because all droplets have a ferromagnetic core, but the ferromagnetic liquid droplets are attracted much more strongly (Fig. 4A and movie S6). As shown in Fig. 4B, using a rotating magnet, the spherical ferromagnetic liquids rotate, whereas the unjammed ferrofluid and CMCjammed ferrofluid droplets do not. The ferromagnetic liquid droplets are also attracted to the center of the magnet, and a dynamically stable pattern forms, balancing a hydrodynamic repulsion against a magnetic attraction, similar to that observed for elastomer discs $(15,16)$ or ferrofluids $(17,18)$ containing MNPs. Similar behavior is seen with ferromagnetic liquid cylinders in which the vortex flow in the oil is visualized with an oil-soluble dye (Fig. 4C and movie S7). The separation distance between the ferromagnetic liquid droplets depends on the rotation velocity (Fig. S5 and movie S8), as expected. The entire patterned assembly of droplets also rotates in response to the rotating field. Upon stopping the rotating magnet, the ferromagnetic liquid cylinders align along the external field direction (movie S9). Absent of a dipole moment, the droplets with and without the jammed CMC monolayer do not spin and move only in a Brownian manner (movie S10). Therefore, the ferromagnetic liquid droplets can be easily separated, rotated in a controlled manner, and patterned (shown in Fig. ${ }_{4} \mathrm{~B}$ ), affording a simple strategy for sorting and spatially arranging the ferromagnetic liquid droplets.

In conclusion, we have demonstrated the transformation of a ferrofluid to a ferromagnetic liquid droplet by the interfacial jamming and magnetization of MNP-surfactants. Ferromagnetic liquid droplets have the fluid characteristics of liquids but the magnetic properties of solids. They can be reconfigured while preserving their magnetic properties, and the attractive/repulsive interactions between ferromagnetic liquid droplets can be manipulated. Separation and patterning of ferromagnetic liquid droplets are easily achieved. The formation of ferromagnetic liquid droplets is reversible, and the interfacial assembly of the MNP-surfactants is responsive to external stimuli and provides systems in which translational and rotational motions can be actuated remotely and precisely by an external magnetic field. 


\section{Acknowledgments}

Funding: This work was supported by the U.S. Department of Energy, Office of Science, Office of Basic Energy Sciences, Materials Sciences and Engineering Division (contract no. DEACo2- 05-CH11231) in the Adaptive Interfacial Assemblies Toward Structuring Liquids program (KCTR16). R.S., A.C., N.K., F.H, and P.F. acknowledge support from U.S. Department of Energy, Office of Science, Office of Basic Energy Sciences, Materials Sciences and Engineering Division (contract no. DE-ACO2-O5-CH11231) in the Non-equilibrium Magnetic Materials program (MSMAG). Work at the Molecular Foundry (AFM imaging) was supported by the Office of Science, Office of Basic Energy Sciences, of the U.S. Department of Energy (contract no. DE-AC02-05CH11231). S.S. was supported by the Beijing NSF (2194083). X.L. was supported by the Beijing Advanced Innovation Center for Soft Matter Science and Engineering at Beijing University of Chemical Technology and China Scholarship Council.

Author contributions: X.L., N.K., R.S., B.A.H., P.D.A., P.F., and T.P.R. made contributions to the conception and design of the experiments. X.L., N.K., A.C., R.S., Y.J., Y.C., J.F., F.H., S.S., and D.W. performed and supported the experiments. X.L., N.K., R.S., P.Y.K., B.A.H., P.D.A., P.F., and T.P.R. interpreted the data and wrote the manuscript.

Competing interests: P.D.A. is also associated with Scuba Probe Technologies.

Data and materials availability: All data are available in the main text or the supplementary materials.

\section{References}

1. S. Odenbach, MRS Bull. 38, 921-924 (2013).

2. R. E. Rosensweig, "Magnetic fluids" in Ferrohydrodynamics (Courier

Corporation, 2013), pp. 34-44.

3. M. Cui, T. Emrick, T. P. Russell, Science 342, 460-463 (2013).

4. Z. Zhang et al., Sci. Adv. 4, eaap8045 (2018).

5. A. H. Lu, E. L. Salabas, F. Schüth, Angew. Chem. Int. Ed. 46, 1222-1244 (2007).

6. J. J. Benkoski et al., J. Am. Chem. Soc. 129, 6291-6297 (2007).

7. R. Streubel et al., Nano Lett. 18, 7428-7434 (2018).

8. E. C. Stoner, E. P. Wohlfarth, Philos. Trans. R. Soc. London A 240, 599-642 (1948).

9. W. Luo, S. R. Nagel, T. F. Rosenbaum, R. E. Rosensweig, Phys. Rev. Lett. 67, 2721-2724 (1991).

10. G. F. Goya, T. S. Berquó, F. C. Fonseca, M. P. Morales, J. Appl. Phys. 94, 35203528 (2003).

11. X. Liu et al., Angew. Chem. Int. Ed. 56, 12594-12598 (2017).

12. J. Forth et al., Adv. Mater. 30, e1707603 (2018).

13. S. Shi et al., Adv. Mater. 30, 1705800 (2018). 
14. A. Toor, S. Lamb, B. A. Helms, T. P. Russell, ACS Nano 12, 2365-2372 (2018).

15. L. Derr, Proc. Am. Acad. Arts Sci. 44, 525-528 (1909).

16. B. A. Grzybowski, H. A. Stone, G. M. Whitesides, Nature 405, 1033-1036 (2000).

17. W. Wang, J. Giltinan, S. Zakharchenko, M. Sitti, Sci. Adv. 3, e16o2522 (2017).

18. J. V. I. Timonen, M. Latikka, L. Leibler, R. H. A. Ras, O. Ikkala, Science 341, 253-257 (2013).

\title{
Supplementary materials
}

\author{
Online: \\ https://science.sciencemag.org/content/365/6450/264/suppl/DC1 \\ Following pages: \\ Materials and Methods \\ Figs. S1 to S5 \\ Table S1 \\ References (19, 20) \\ Attached to this archive record: \\ Movies S1 to S10
}

\begin{abstract}
Reconfigurable ferromagnetic liquid droplets
Xubo Liu, Noah Kent, Alejandro Ceballos, Robert Streubel, Yufeng Jiang, Yu Chai, Paul Y. Kim, Joe Forth, Frances Hellman, Shaowei Shi, Dong Wang, Brett A. Helms, Paul D. Ashby, Peter Fischer, \& Thomas P. Russell

Science, 365 (6450), • DOI: 10.1126/science.aaw8719

Liquid reconfigurable ferromagnetic materials - Ferromagnetic materials show a permanent magnetic dipole, whereas superparamagnetic ones only show magnetic properties under an applied field. Some materials, like ferrofluids, show liquid-like behavior but do not retain their magnetization in the absence of an applied field. Liu et al. show remnant magnetization of otherwise superparamagnetic magnetite nanoparticles at an oil-water interface of emulsion droplets (see the Perspective by Dreyfus). The permanent magnetization could be controlled by coupling and uncoupling the magnetization of individual nanoparticles, making it possible to "write and erase" shapes of the droplets or to elongate them into cylinders.
\end{abstract}




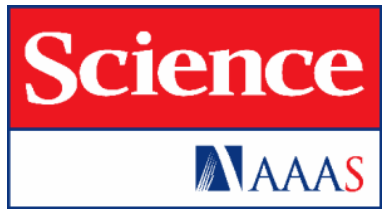

\section{Supplementary Materials for}

\section{Reconfigurable ferromagnetic liquid droplets}

Xubo Liu, Noah Kent, Alejandro Ceballos, Robert Streubel, Yufeng Jiang, Yu Chai, Paul Y. Kim, Joe Forth, Frances Hellman, Shaowei Shi, Dong Wang, Brett A. Helms, Paul D. Ashby, Peter Fischer, Thomas P. Russell*

*Corresponding author. Email: tom.p.russell@ gmail.com Published 19 July 2019, Science 365, 264 (2019) DOI: $10.1126 /$ science.aaw8719

This PDF file includes:

Materials and Methods

Figs. S1 to S5

Table S1

Captions for Movies S1 to S10

References

Other Supplementary Material for this manuscript includes the following: (available at science.sciencemag.org/content/365/6450/264/suppl/DC1)

Movies S1 to S10 


\section{Materials and Methods}

Chemicals

The following chemicals were used as received from Sigma-Aldrich: Carboxyl-modified iron oxide nanoparticles, $\left(\mathrm{Fe}_{3} \mathrm{O}_{4}-\mathrm{CO}_{2} \mathrm{H} \mathrm{NPs}, 30 \mathrm{~nm}\right)$. The iron oxide core with $22 \mathrm{~nm}$ diameter was coated with a $4 \mathrm{~nm}$ thin layer of polymer ligands with carboxyl-end-functionalized, forming a periphery of charged units that stabilizes the NPs dispersion in water; Polyethylene glycol coated iron oxide nanoparticles, $\left(\mathrm{Fe}_{3} \mathrm{O}_{4}-\mathrm{PEG} \mathrm{NPs}, 30 \mathrm{~nm}\right)$; Amine-modified polyhedral oligomeric silsesquioxane (POSS-NH $2, M_{w}=917 \mathrm{~g} \mathrm{~mol}^{-1}$ ), Toluene $(>99.8 \%)$; Carbon tetrachloride ( $>$ 99.9\%); Hydrochloric acid (37\%); Nile Red (>98\%); Fluorescein sodium salt (acid yellow 73, $M_{w}$

$\left.10=376 \mathrm{~g} \mathrm{~mol}^{-1}\right)$; Rhodamine B (>95\%); Sodium carboxymethyl cellulose $\left(\mathrm{CMC}-\mathrm{CO}_{2} \mathrm{Na}, M_{w}=\sim 90\right.$ $\left.\mathrm{Kg} \mathrm{mol}^{-1}\right)$.

\section{Magnetism characterization}

A vibrating sample magnetometer (Lakeshore) was used to measure the M-H hysteresis loop

15 of the liquid droplets at room temperature (shown in Fig. S2). For the jamming state of single droplets, the droplet containing a dispersion of $\mathrm{MNPs}\left(1,5\right.$ and $9 \mu \mathrm{L}$ respectively, $\mathrm{pH} 4.5,\left[\mathrm{Fe}_{3} \mathrm{O}_{4}\right.$ $\left.\left.\mathrm{CO}_{2} \mathrm{H} \mathrm{MNPs}\right]=0.5 \mathrm{~g} \mathrm{~L}^{-1}\right)$ was immersed in $\sim 50 \mu \mathrm{L}$ of pure toluene $\left(\left[\mathrm{POSS}-\mathrm{NH}_{2}\right]=1.0 \mathrm{~g} \mathrm{~L}^{-1}\right)$ and sealed in a liquid sample holder (730935 Kel-F liquid disposable cup, Lakeshore) for measurements within $2 \mathrm{hr}$. For the jamming state of emulsified droplets with volume of 5 and 9

$20 \mu \mathrm{L}$, the droplet containing MNPs $\left(\mathrm{pH} 4.5,\left[\mathrm{Fe}_{3} \mathrm{O}_{4}-\mathrm{CO}_{2} \mathrm{H}\right.\right.$ MNPs $\left.]=0.5 \mathrm{~g} \mathrm{~L}^{-1}\right)$ was sealed in the holder and then emulsified by ultrasonication for $10 \mathrm{~s}$. For the unjamming state, $\mathrm{pH}$ of the droplets was tuned to 4.5, 7.0, and 9.5 at MNPs concentration of $0.5 \mathrm{~g} \mathrm{~L}^{-1}$, and $\mathrm{pH} 4.5$ at MNPs concentration of $0.05 \mathrm{~g} \mathrm{~L}^{-1}$, all of those droplets immersed in the toluene containing $0.01 \mathrm{~g} \mathrm{~L}^{-1}$ of POSS $-\mathrm{NH}_{2}$, by which the droplet surface cannot reach fully coverage state during the vibrating

25 sample magnetometer measurements.

Measurement of interfacial tensions and surface coverages

An example of the interfacial tension between water and toluene measured with a tensiometer (KRÜSS GmbH, DSA30) is shown in Fig. S1A, B. The surface coverage $C_{\mathrm{s}}=S_{\text {wrinkling }} / S_{\text {free }}$ of the

30 MNPs-surfactants at the water/toluene interface was estimated using a pendant drop from the initial volume (surface area, $S_{\text {free}}$ ) and from the volume of the droplet (surface area, $S_{\text {wrinkling }}$ ) at which the assembly of the MNP-surfactants jammed and began to wrinkle. The droplet is assumed to be rotationally symmetric. The results are shown in Fig. S1C. The higher the surface coverage, the less the droplet volume must be reduced before wrinkling is observed.

AFM imaging

An atomic force microscope (AFM, Bruker, Icon) was used to measure the diameter and morphology of MNPs assembled at the water/toluene interface. The assembled film at the water/toluene interface when jamming occurred was retrieved from the liquid interface using a clean silicon wafer and dried at ambient conditions. The AFM image shown in Fig. S4B indicates that the diameter of MNPs at the interface is $\sim 30 \mathrm{~nm}$.

\section{TEM imaging}

We transferred a monolayer of jammed NPs to a silicon nitride wafer for TEM imaging. From the TEM image, the particles are $\sim 30 \mathrm{~nm}$. Some particles are quite anisotropic in shape. Depending on the angle between the lattice of the iron oxide atoms and the electron beam, the contrast of the 
particles will vary. The iron oxide core is also wrapped by a $3 \sim 4 \mathrm{~nm}$ layer of carboxyl endfunctionalized polyetheleneglycol (PEG). The in-surface shape anisotropy leads to disordered packing structure. The nearest-neighbor numbers typically varying from 5 to 8 . Images were taken at room temperature using full-field TEM mode with an acceleration voltage of $300 \mathrm{kV}$ (TEAM$1)$.

\section{Fabrication of liquid cylinders}

Using all-liquid 3D printing in a microfluidic device, we continuously printed all-liquid cylinders of the FLD. Upon printing, a slight decrease in the interfacial area jams the MNPsurfactants that form and assemble at the interface. In particular, $2 \mathrm{~mm}$ long liquid cylinders with a diameter of $1 \mathrm{~mm}$ were printed through a $1 \mathrm{~mm}$ diameter PTFE tube, as shown in Fig. S3, by setting a flow rate of the continuous oil phase containing ligands, $Q_{\mathrm{O}}=30 \mu \mathrm{L} \mathrm{min}^{-1}$, and that of the aqueous dispersion of $\mathrm{Fe}_{3} \mathrm{O}_{4}-\mathrm{CO}_{2} \mathrm{H}$ MNPs, $Q_{\mathrm{W}}=90 \mu \mathrm{L} \mathrm{min}^{-1}$, respectively. The shape of FLD droplet changes from spherical to cylindrical when $Q_{\mathrm{W}} / Q_{\mathrm{O}}>1$. If $Q_{\mathrm{W}} / Q_{\mathrm{O}}$ is large enough, a continuous filament of the aqueous phase can be printed in the oil phase. Within the capillary, the interface of droplets from spherical to cylindrical shape undergo extensive plastic deformation, with the droplet's shape governed by both surface wetting properties of the droplet against the glass or PTFE pipette and the complex mechanical properties of the disordered MNPS assembly $(19,20)$.

\section{Fabrication of electromagnetic solenoid}

An aluminum wire with $\sim 0.2 \mathrm{~mm}$ diameter was wound into a solenoid to generate currentinduced magnetic fields that interact with the FLDs. The setup provided a magnetic field of up to $1 \sim 2 \mathrm{kA} \mathrm{m}^{-1}$ at the center of the solenoid using a current of $I=2 \mathrm{~A}$ and $N=15$ windings, each

25 separated by $L=1 \sim 2 \mathrm{~mm}$. The measured value is in good agreement with the analytical value of $\mathrm{H}=I / L=1 \sim 2 \mathrm{kA} \mathrm{m}^{-1}$.

Fabrication of density gradient oil phase

The solubility of toluene and $\mathrm{CCl}_{4}$ in water are $0.051 \% \mathrm{w} / \mathrm{w}$ and $0.08 \% \mathrm{w} / \mathrm{w}$, and the dynamic viscosity of toluene and $\mathrm{CCl}_{4}$ at $20{ }^{\circ} \mathrm{C}$ are $0.59 \mathrm{cp}$ and $0.97 \mathrm{cp}$, respectively. They are immiscible with water. Toluene $\left(0.865 \mathrm{~g} \mathrm{~cm}^{-3}\right)$ is able to segregate and form a thin layer on top of $\mathrm{CCl}_{4}$ phase $\left(1.595 \mathrm{~g} \mathrm{~cm}^{-3}\right)$ without fierce shaking, but will form a stable transition layer with a gradient density from $0.865 \mathrm{~g} \mathrm{~cm}^{-3}$ to $1.595 \mathrm{~g} \mathrm{~cm}^{-3}$ at room temperature due to the oil miscibility (see Fig. S4A). This enables the buoyant of water droplets $\left(\sim 1.0 \mathrm{~g} \mathrm{~cm}^{-3}\right)$ at grade, where volume ratio of toluene 35 to $\mathrm{CCl}_{4}$ is $4: 1$ and the dynamic viscosity is $\sim 6.66 \times 10^{-4} \mathrm{~kg} \mathrm{~m}^{-1} \mathrm{~s}^{-1}$, while immersing in the oil phase. All the spinning FLDs were imaged in the gradient oil using an optical microscope. 
Figures and Tables
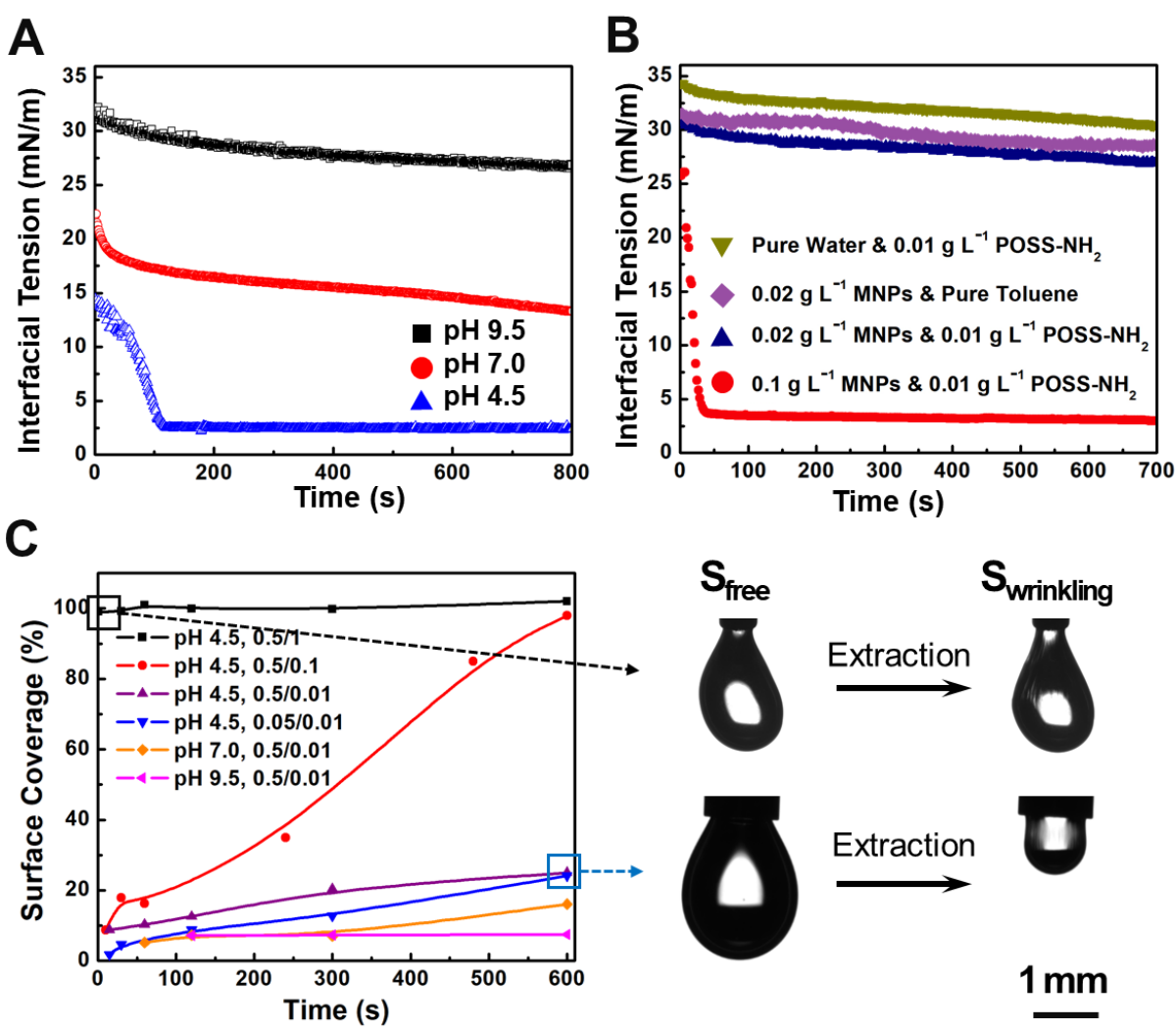

Fig. S1. Temporal evolution of interfacial tension of droplets decorated with MNPs. (A) The influence of $\mathrm{pH}$ on interfacial tension is shown for $\mathrm{Fe}_{3} \mathrm{O}_{4}-\mathrm{CO}_{2} \mathrm{H}$ MNPs $\left(0.5 \mathrm{~g} \mathrm{~L}^{-1}\right)$ with POSS-

$5 \quad \mathrm{NH}_{2}\left(0.01 \mathrm{~g} \mathrm{~L}^{-1}\right)$. (B) Effect of MNPs concentration and respective jamming on interfacial tension for $\mathrm{pH} 4.5$, [POSS-NH 2$]=0.01 \mathrm{~g} \mathrm{~L}^{-1}$, and $\left[\mathrm{Fe}_{3} \mathrm{O}_{4}-\mathrm{CO}_{2} \mathrm{H} \mathrm{MNPs}\right]=0.02$ and $0.1 \mathrm{~g} \mathrm{~L}^{-1}$. (C) Surface coverage of MNPs-surfactants at the water/toluene interface as a function of time for $\mathrm{pH} 4.5,7.0$, and 9.5, $\left[\mathrm{Fe}_{3} \mathrm{O}_{4}-\mathrm{CO}_{2} \mathrm{H} \mathrm{MNPs}\right]=0.5$ or $0.05 \mathrm{~g} \mathrm{~L}^{-1}$, and $\left[\mathrm{POSS}-\mathrm{NH}_{2}\right]=0.01,0.1$ or $1 \mathrm{~g} \mathrm{~L}^{-1}$, respectively. Surface coverage, $C_{\mathrm{s}}$, is defined as the ratio of surface area at MNPs jamming and free state, respectively, $C_{\mathrm{s}}=S_{\text {wrinkling }} / S_{\text {free. }}$. 
A

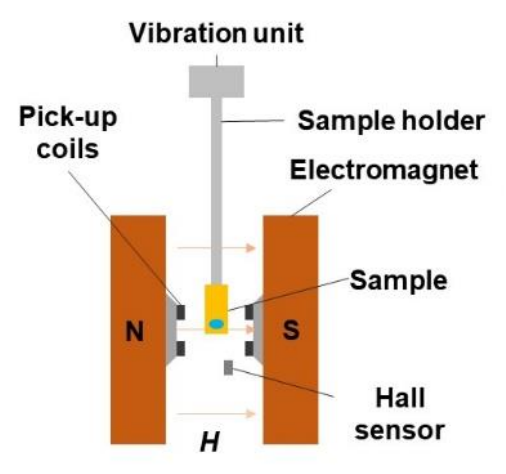

B

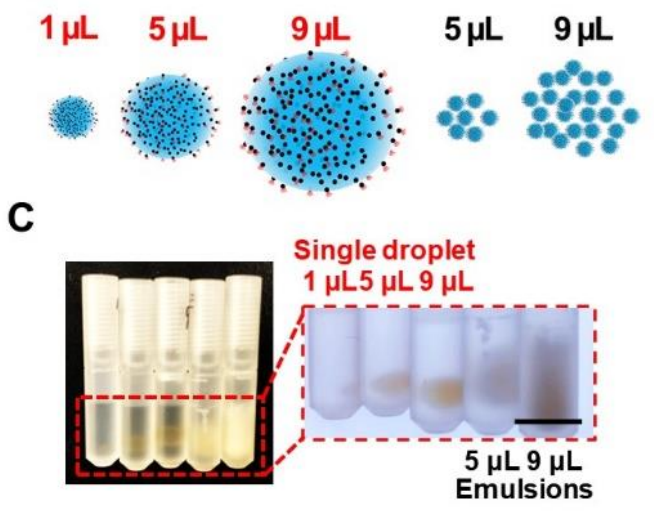

D

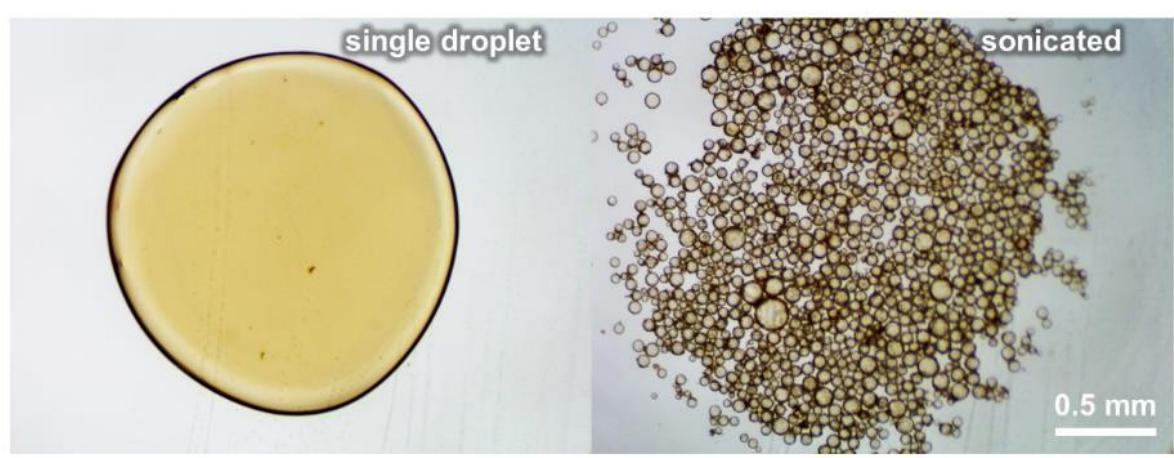

Fig. S2. Vibrating sample magnetometer measurement and liquid sample preparation. (A) Schematics of vibrating sample magnetometer for hysteresis loop measurement. (B) Schematics and (C) images of single droplets with $1 \mu \mathrm{L}, 5 \mu \mathrm{L}, 9 \mu \mathrm{L}$ and emulsions of sonicated droplets with $5 \mu \mathrm{L}, 9 \mu \mathrm{L}$ after being ultrasonicated for $10 \mathrm{~s}$, with the surface to volume ratio, $S / V$, increasing $\sim 2$ orders in magnitude. Scale bars, $5 \mathrm{~mm}$. (D) $1-\mu \mathrm{L}$ droplet with a diameter of $\sim 1.5 \mathrm{~mm}$ when spread on a glass microscope slide for observation, the mean size of the droplet is $\sim 50 \mu \mathrm{m}$ after sonication. Scale bar: $0.5 \mathrm{~mm}$. 


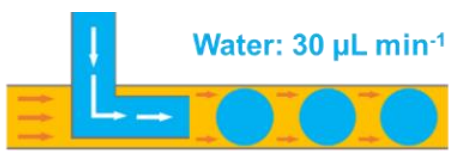

Oil: $90 \mu \mathrm{L}$ min $^{-1}$

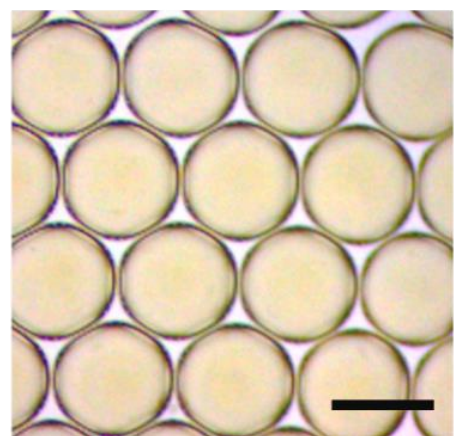

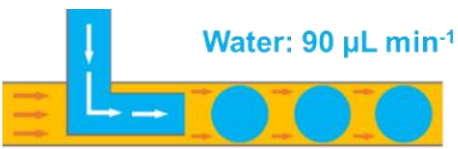

Oil: $90 \mu \mathrm{L} \mathrm{min}^{-1}$

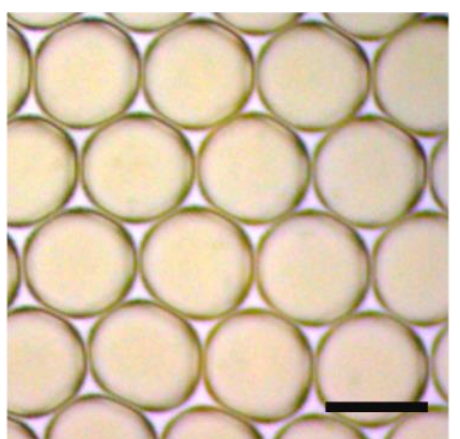

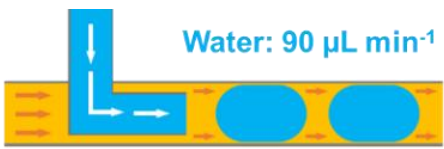

Oil: $30 \mu \mathrm{L} \min ^{-1}$

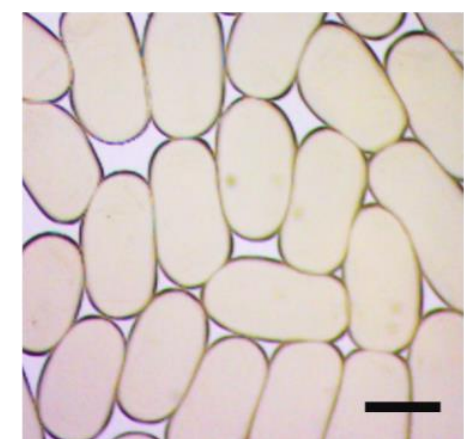

Fig. S3. Shaping droplets by all-liquid 3D printing in microfluidic devices. Shape of the droplets can be optimized by changing diameter and length of PTFE tube, where oil and water mix and flow, and pump-controlled flow rate of water and oil phases. $\left[\mathrm{Fe}_{3} \mathrm{O}_{4}-\mathrm{CO}_{2} \mathrm{H}\right.$ MNPs in water] $=$ $0.5 \mathrm{~g} \mathrm{~L}^{-1}, \mathrm{pH} 4.5$; [POSS-NH 2 in toluene] $=10 \mathrm{~g} \mathrm{~L}^{-1}$. The printed droplets can be collected using petri dish. Scale bars: $1 \mathrm{~mm}$. 
A

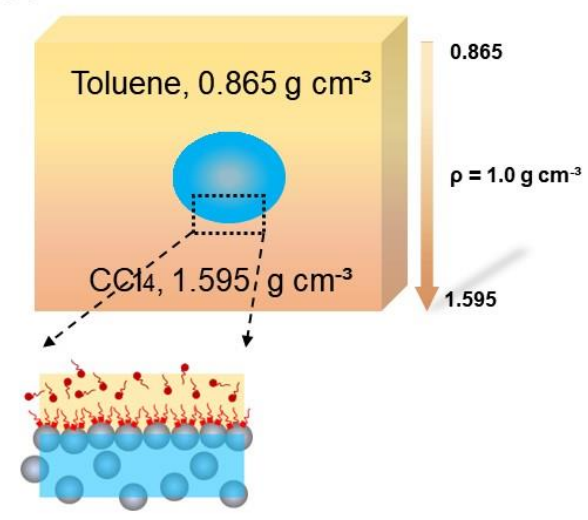

$30 \mathrm{~nm}, \mathrm{Fe} 3 \mathrm{O} 4-\mathrm{COOH} \mathrm{NPs}$
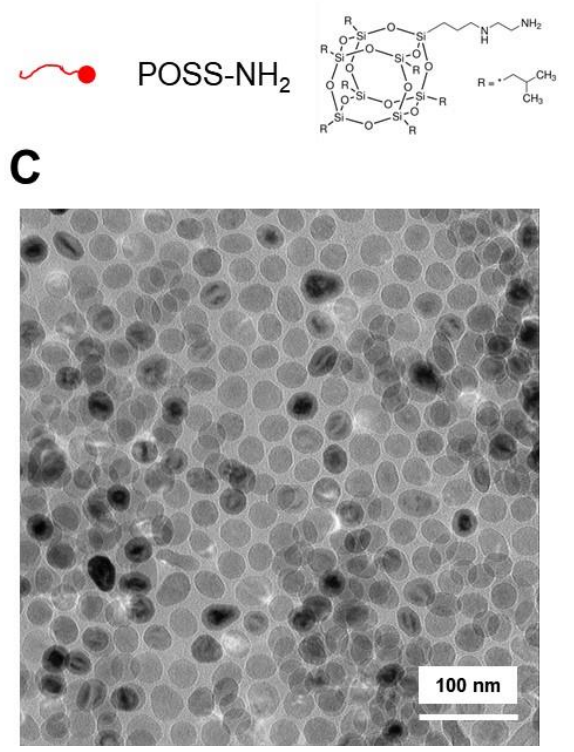

B

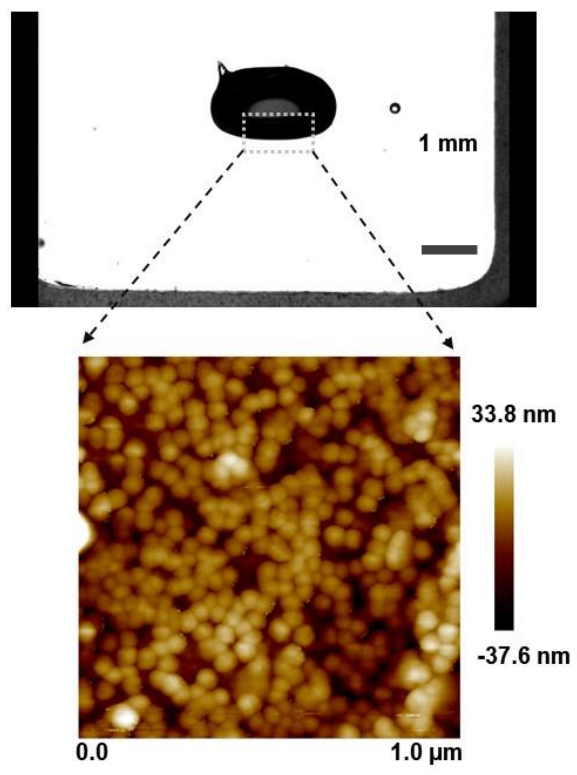

Fig. S4. A droplet buoyant in the gradient oil and morphology of MNPs at interfaces. (A) carboxylated iron oxide nanoparticles $\left(\mathrm{Fe}_{3} \mathrm{O}_{4}-\mathrm{CO}_{2} \mathrm{H}\right.$ MNPs) assemble with amine functionalized POSS (POSS-NH $)$ at the water/toluene interface. The aqueous droplet $\left(1.0 \mathrm{~g} \mathrm{~cm}^{-3}\right)$ is buoyant in the bilayer oil mixture of toluene and $\mathrm{CCl}_{4}(\mathrm{v} / \mathrm{v}=4: 1)$ with a density gradient without any fierce shaking. (B) Side-view backlight image of buoyant droplet, stable in the non-spherical shape, indicates that MNPs-surfactants jam at water/oil interfaces very well. The AFM image of the assembled film indicates the jammed state of MNPs at liquid interfaces. The size of nanoparticles is $\sim 30 \mathrm{~nm}$. (C) TEM image of jammed MNPs transferred from water-toluene interface to silicon nitride wafer in the dry state. 


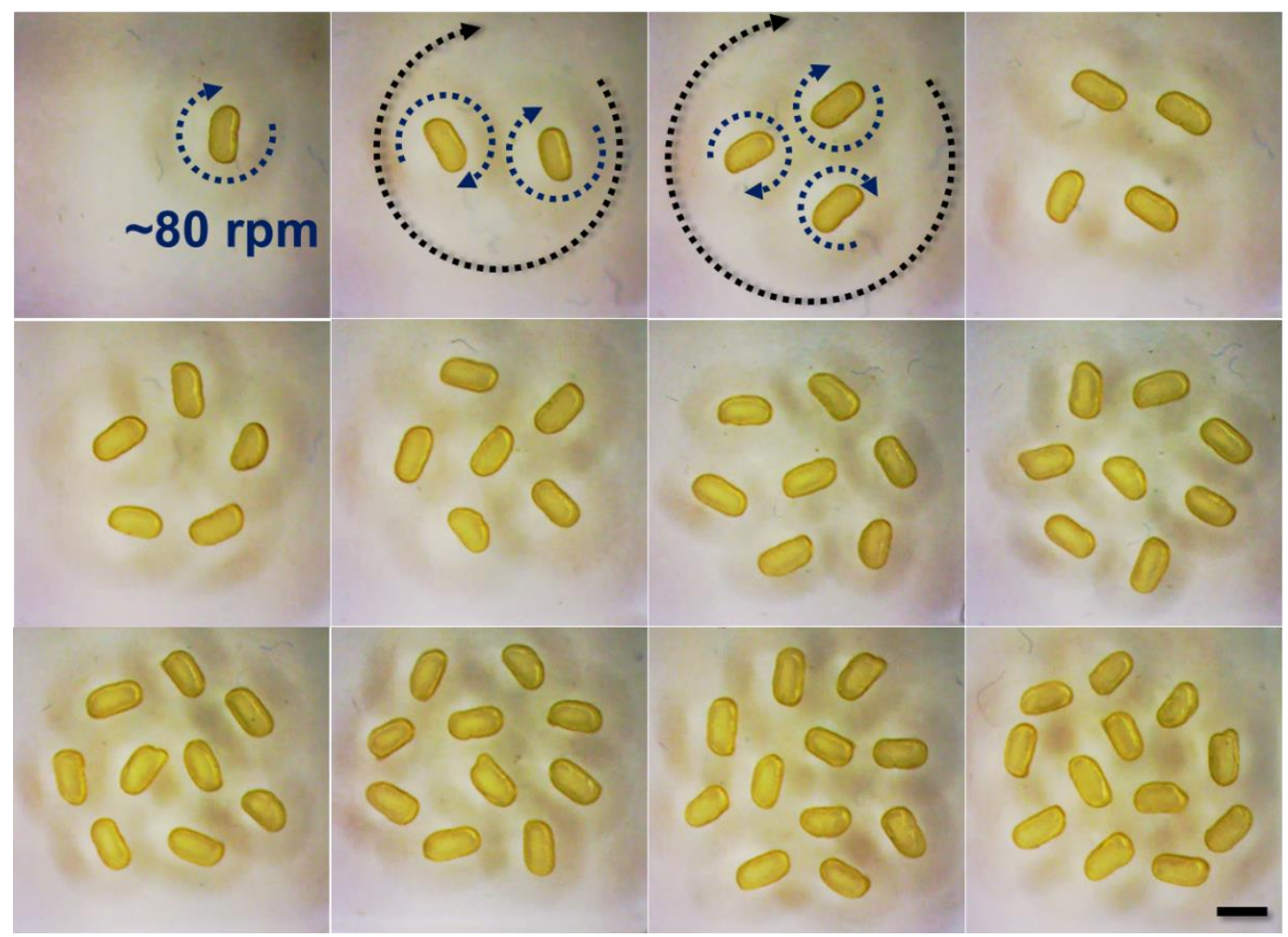

Fig. S5. Top view of stable patterns formed by rotating cylindrical ferromagnetic liquid droplets. Dynamic spinning patterns depend on the droplet numbers (from 1 to 12) and the rotating speed $(300 \mathrm{rpm})$ of the magnet underneath, which generate a rotating magnetic field with a specific strength $\left(\mu_{0} \mathrm{M}=1.25 \mathrm{~T}\right.$ at surface of the bar magnet $)$. Scale bar: $2 \mathrm{~mm}$. 


\begin{tabular}{|c|c|c|c|c|c|}
\hline & $\begin{array}{c}\text { Volume } \\
(\mu \mathrm{L})\end{array}$ & $\begin{array}{c}\text { Saturation } \\
\text { Magnetization, } M_{\mathrm{s}} \\
\left(\times 10^{-8} \mathrm{~A} \mathrm{~m}^{2}\right)\end{array}$ & $\begin{array}{c}\text { Remanent } \\
\text { Magnetization, } M_{\mathrm{r}} \\
\left(\times 10^{-8} \mathrm{~A} \mathrm{~m}^{2}\right)\end{array}$ & $\begin{array}{c}\text { Coercivity, } H_{\mathrm{c}} \\
\left(\mathrm{kA} \mathrm{m}^{-1}\right)\end{array}$ & $M_{\mathrm{r}} / M_{\mathrm{s}}$ \\
\hline \multirow{3}{*}{$\begin{array}{c}\text { Single } \\
\text { Droplet }\end{array}$} & 1 & 1.56 & 0.48 & 6.4 & 0.31 \\
\cline { 2 - 6 } & 5 & 7.86 & 1.89 & 7.2 & 0.24 \\
\cline { 2 - 6 } & 9 & 15.6 & 4.15 & 7.2 & 0.27 \\
\hline \multirow{3}{*}{ Emulsion } & 5 & 7.85 & 1.76 & 6.0 & 0.22 \\
\cline { 2 - 6 } & 9 & 14.4 & 3.70 & 6.4 & 0.26 \\
\hline
\end{tabular}

Table S1. Values of saturation magnetization, remanent magnetization, coercive field, and $M_{\mathrm{r}} / M_{\mathrm{s}}$ calculated according to the hysteresis loops. 


\section{Movie S1.}

A non-magnetized cylindrical ferromagnetic liquid droplet buoyant in the oil is attracted into the solenoid once magnetic field is applied. We observe that the printed FLD cylinder enter into the solenoid with an acceleration after a current is applied to the solenoid to generate a gradient magnetic field. Magnetization happens at first then the attraction makes effect. The video is played with 4 times speed up. The length of the FLD cylinder is $2 \mathrm{~mm}$.

\section{Movie S2.}

A magnetized cylindrical ferromagnetic liquid droplet buoyant in the oil is attracted into the solenoid faster. The magnetized FLD cylinder interacts with solenoid by north-south attraction initially, and reaches to the solenoid faster without the magnetization process. The video is played with 4 times speed up. The length of the FLD cylinder is $2 \mathrm{~mm}$.

\section{Movie S3.}

A magnetized cylindrical ferromagnetic liquid droplet buoyant in the oil is attracted into the solenoid slower. The repositioned magnetized FLD cylinder rotates to align with the field flux at

15 first due to the south-south repulsion, and then reaches to the solenoid under the north-south attraction. The dipole moment of the FLD cylinder is fixed very well by the interfacial jammed magnetic nanoparticle surfactants. The video is played with 4 times speed up. The length of the FLD cylinder is $2 \mathrm{~mm}$.

\section{Movie S4.}

20 Magnetic N-S dipole interactions between two FLDs. Two magnetized liquid cylinders buoyant in the oil attract each other by coordinated actions of north-south dipole attraction and north-north, south-south repulsions. The video is played at 6 times the actual speed. The volume of the FLD droplets is $2 \mu \mathrm{L}$ and the length of the FLD cylinder is $2 \mathrm{~mm}$.

\section{Movie S5.}

25 Deformation of spherical ferromagnetic liquid droplet into cylindrical shape. A spherical ferromagnetic liquid droplet was drawn into the glass capillary and deformed into a cylindrical shape. The reshaped ferromagnetic liquid droplet was able to rotate, following with the external spinning magnetic field. The video is played at 3 times actual speed. The volume of the ferromagnetic liquid droplet is $2 \mu \mathrm{L}$ and the length of the ferromagnetic liquid cylinder is $2 \mathrm{~mm}$.

\section{Movie S6.}

Ferromagnetic liquid droplets are separated from the other paramagnetic liquid droplets by static permanent magnet. The brown spherical FLD droplets with MNPSs jammed at water/oil interfaces move faster after being magnetized than the FF droplets. Separation from the other magnetic droplets is well controlled by external static and rotating magnetic field. The video is

35 played with 2 times speed up. The diameter of droplets is $1 \mathrm{~mm}$.

\section{Movie S7.}

A drop of Nile Red toluene solution is added into the gradient oil phase to visualize the flow fields around vortex-generating FLDs. We observe the hydrodynamic radius of a spinning FLD cylinder clearly. The clockwise fluid flow around every FLD s generated vortex-vortex repulsion, 
leading to the dynamic pattern formation in couple with the magnetic attractions from the rotating magnet. The video is in real time. Scale bar, $2 \mathrm{~mm}$.

\section{Movie S8.}

A dynamic pattern is formed by 12 rotating FLD cylinders buoyant in the surrounding oil

5 phase. These patterns are similar with those of Mayer's floating magnets. The attraction to the center of bar magnet and the repulsions between each FLD s result in the special pattern where each FLD stays in the same position relatively. The video is in real time. The length of the FLD cylinder is $2 \mathrm{~mm}$

\section{Movie S9.}

10 Manipulating the orientation of FLD cylinders with an external magnetic field generated by a bar magnet. All magnetized liquid cylinders are aligned along the direction of flow field. They can re-orient immediately along with the changing direction of external magnetic field. The video is in real time. The length of the ferromagnetic liquid cylinder is $2 \mathrm{~mm}$.

\section{Movie S10.}

15 Ferromagnetic liquid droplets are separated from the other paramagnetic liquid droplets by rotating permanent magnet. The spherical FLD droplets with dipole moment rotate along with the rotating magnetic field and form patterns due to the hydrodynamic repulsion and magnetic attraction. The red and fluorescent green paramagnetic droplets without dipole moment only move along with the rotating field randomly, and are expelled from the central area by the vortex flow. The video is played with 6 times speed up. The diameter of droplets is $1 \mathrm{~mm}$. 


\section{References and Notes}

1. S. Odenbach, Ferrofluids and their applications. MRS Bull. 38, 921-924 (2013).

2. R. E. Rosensweig, "Magnetic fluids" in Ferrohydrodynamics (Courier Corporation, 2013), pp. 34-44.

3. M. Cui, T. Emrick, T. P. Russell, Stabilizing liquid drops in nonequilibrium shapes by the interfacial jamming of nanoparticles. Science 342, 460-463 (2013).

4. Z. Zhang, Y. Jiang, C. Huang, Y. Chai, E. Goldfine, F. Liu, W. Feng, J. Forth, T. E. Williams, P. D. Ashby, T. P. Russell, B. A. Helms, Guiding kinetic trajectories between jammed and unjammed states in 2D colloidal nanocrystal-polymer assemblies with zwitterionic ligands. Sci. Adv. 4, p8045 (2018).

5. A. H. Lu, E. L. Salabas, F. Schüth, Magnetic nanoparticles: Synthesis, protection, functionalization, and application. Angew. Chem. Int. Ed. 46, 1222-1244 (2007).

6. J. J. Benkoski, S. E. Bowles, B. D. Korth, R. L. Jones, J. F. Douglas, A. Karim, J. Pyun, Field induced formation of mesoscopic polymer chains from functional ferromagnetic colloids. J. Am. Chem. Soc. 129, 6291-6297 (2007).

7. R. Streubel, N. Kent, S. Dhuey, A. Scholl, S. Kevan, P. Fischer, Spatial and temporal correlations of XY macro spins. Nano Lett. 18, 7428-7434 (2018).

8. E. C. Stoner, E. P. Wohlfarth, A mechanism of magnetic hysteresis in heterogeneous alloys. Philos. Trans. R. Soc. Lond. A 240, 599-642 (1948).

9. W. Luo, S. R. Nagel, T. F. Rosenbaum, R. E. Rosensweig, Dipole interactions with random anisotropy in a frozen ferrofluid. Phys. Rev. Lett. 67, 2721-2724 (1991).

10. G. F. Goya, T. S. Berquó, F. C. Fonseca, M. P. Morales, Static and dynamic magnetic properties of spherical magnetite nanoparticles. J. Appl. Phys. 94, 3520-3528 (2003).

11. X. Liu, S. Shi, Y. Li, J. Forth, D. Wang, T. P. Russell, Liquid tubule formation and stabilization using cellulose nanocrystal surfactants. Angew. Chem. Int. Ed. 56, 1259412598 (2017).

12. J. Forth, X. Liu, J. Hasnain, A. Toor, K. Miszta, S. Shi, P. L. Geissler, T. Emrick, B. A. Helms, T. P. Russell, Reconfigurable printed liquids. Adv. Mater. 30, e1707603 (2018).

13. S. Shi, X. Liu, Y. Li, X. Wu, D. Wang, J. Forth, T. P. Russell, Liquid Letters. Adv. Mater. 30, 1705800 (2018).

14. A. Toor, S. Lamb, B. A. Helms, T. P. Russell, Reconfigurable microfluidic droplets stabilized by nanoparticle surfactants. ACS Nano 12, 2365-2372 (2018).

15. L. Derr, A photographic study of mayer's floating magnets. Proc. Am. Acad. Arts Sci. 44, 525-528 (1909).

16. B. A. Grzybowski, H. A. Stone, G. M. Whitesides, Dynamic self-assembly of magnetized, millimetre-sized objects rotating at a liquid-air interface. Nature 405, 1033-1036 (2000).

17. W. Wang, J. Giltinan, S. Zakharchenko, M. Sitti, Dynamic and programmable self-assembly of micro-rafts at the air-water interface. Sci. Adv. 3, e1602522 (2017). 
18. J. V. I. Timonen, M. Latikka, L. Leibler, R. H. A. Ras, O. Ikkala, Switchable static and dynamic self-assembly of magnetic droplets on superhydrophobic surfaces. Science 341, 253-257 (2013).

19. N. C. Keim, P. E. Arratia, Mechanical and microscopic properties of the reversible plastic regime in a 2D jammed material. Phys. Rev. Lett. 112, 028302 (2014).

20. H. Xu, S. Melle, K. Golemanov, G. Fuller, Shape and buckling transitions in solid-stabilized drops. Langmuir 21, 10016-10020 (2005). 